\title{
Neural dynamics of selective attention deficits in HIV-associated neurocognitive disorder
}

Brandon J. Lew, Timothy J. McDermott, Alex I. Wiesman, Jennifer O'Neill, RN, Mackenzie S. Mills, Kevin R. Robertson, PhD, Howard S. Fox, MD, PhD, Susan Swindells, MBBS, and Tony W. Wilson, PhD

Neurology ${ }^{\circledR}$ 2018;91:e1860-e1869. doi:10.1212/WNL.0000000000006504

\author{
Correspondence \\ Dr. Wilson \\ twwilson@unmc.edu
}

\section{Abstract \\ Objective}

To identify the neural markers of attention dysfunction in patients with HIV-associated neurocognitive disorder (HAND).

\section{Methods}

Sixty participants, including 40 HIV-infected adults (half with HAND) and 20 demographically matched controls performed a visual selective attention task while undergoing high-density magnetoencephalography. Neuronal activity related to selective attention processing was quantified and compared across the 3 groups, and correlated with neuropsychological measures of attention and executive function. Spontaneous neural activity was also extracted from these attention-related cortical areas and examined with respect to HAND status.

\section{Results}

HIV-infected participants with and without HAND exhibited behavioral selective attention deficits on the magnetoencephalography task, as indicated by an increased flanker effect. Neuronal measures of flanker interference activity in the alpha and theta range revealed differential dynamics in attention-related brain areas across the 3 groups, especially in those with HAND. In addition, theta range flanker interference activity in the left inferior frontal and dorsolateral prefrontal cortex was associated with executive function and attention composite scores, respectively. Progressively stronger spontaneous alpha and theta activity was also found in unimpaired HIV-infected and HAND participants relative to controls across brain regions implicated in different components of attention processing.

\section{Conclusions}

Behavioral and neuronal metrics of selective attention performance distinguish participants with HAND from controls and unimpaired HIV-infected participants. These metrics, along with measures of local spontaneous neural activity, may hold promise as early markers of cognitive decline in participants with HIV infection and be useful prognostic indicators for HAND.

From the Departments of Neurological Sciences (B.J.L., T.J.M., A.I.W., M.S.M., T.W.W.), Internal Medicine (J.O., S.S.), and Pharmacology and Experimental Neuroscience (H.S.F.), University of Nebraska Medical Center, Omaha; and Department of Neurology (K.R.R.), University of North Carolina School of Medicine, Chapel Hill.

Go to Neurology.org/N for full disclosures. Funding information and disclosures deemed relevant by the authors, if any, are provided at the end of the article. 


\section{Glossary}

$\mathrm{ADL}=$ activities of daily living; ANI = asymptomatic neurocognitive impairment; ANOVA $=$ analysis of variance; cART = combination antiretroviral therapy; DLPFC = dorsolateral prefrontal cortex; HAD = HIV-associated dementia; HAND = HIVassociated neurocognitive disorder; $\mathbf{M E G}$ = magnetoencephalography; $\mathbf{M N D}=$ mild neurocognitive disorder.

Neurocognitive deficits in attention and executive functioning are defining aspects of HIV-associated neurocognitive disorders (HAND). ${ }^{1-3}$ These symptoms are often more subtle in the combination antiretroviral therapy (cART) era, and therefore a more specific neural marker of HAND would be of major value to better identify and care for these patients, and to further understanding of the neurobiology. ${ }^{4}$

Neural aberrations have been shown in HIV-infected adults, particularly in brain areas serving attention and executive function processing such as prefrontal, parietal, and visual cortices. ${ }^{5-10}$ The relationship of these aberrations to HAND is not fully understood, and no study to date has shown that activity in higher-order association cortices can distinguish between HIV-infected participants with and without HAND.

Using state-of-the-art magnetoencephalography (MEG), we evaluated the neural dynamics serving selective attention processing in HIV-infected participants with and without HAND, and a demographically matched uninfected group. We hypothesized that HIV-infected participants would have neural aberrations in parietal and prefrontal regions involved in selective attention function, along with behavioral attention impairments, and that these deficits would be significantly more severe in those with HAND.

\section{Methods}

\section{Participants}

Twenty uninfected adult controls, 20 unimpaired HIVinfected adults, and 20 adults with HAND participated in the study. To minimize the effect of aging, participants were aged 30 to 60 years at enrollment (mean $=44.05 ; \mathrm{SD}=7.44$ ). The 3 groups were matched on age, sex, education, handedness, and ethnicity. The HAND group comprised 3 severity subtypes based on the Frascati criteria. ${ }^{1}$ Group demographics and neuropsychological profiles are reported in the table. All participants with HIV infection were being treated with cART, and all but 2 had undetectable viremia $(<20$ copies $/ \mathrm{mL})$. Further participant information is available in data available from Dryad (appendix e-1, doi.org/10.5061/dryad.1m6764d).

\section{Standard protocol approvals, registrations, and patient consents}

The institutional review board at the University of Nebraska Medical Center approved this investigation. Each participant provided written informed consent following a detailed explanation of the study. All participants completed the same protocol.

\section{Neuropsychological testing}

Participants completed a battery of neuropsychological assessments that were selected based on recommendations of the Frascati consensus. ${ }^{1}$ This battery assessed multiple functional domains: executive functioning (verbal fluency, semantic fluency, Stroop interference, and Trail Making Part B), attention (Symbol Search, Stroop word), speed of processing (Trail Making Part A, digit symbol, Stroop color), fine motor (grooved pegboard), verbal learning and memory (Hopkins Verbal Learning Test-Revised), and language (Wide Range Achievement Test 4 reading). Each participant's scores on each test were converted to demographically adjusted $z$ scores using published normative data, and composite scores for each domain were computed by averaging the $z$ scores across the tests included in a specific domain. ${ }^{11}$ Using these assessments and activities of daily living (ADL), patients were diagnosed with HAND according to the Frascati guidelines. Generally, HIV-infected patients were categorized as having no cognitive impairment, asymptomatic neurocognitive impairment (ANI) (i.e., having at least 2 cognitive domains 1 SD below the standardized mean, with no ADL deficits), mild neurocognitive disorder (MND) (i.e., at least 2 cognitive domains 1 SD below the standardized mean, with ADL deficits), or HIV-associated dementia (HAD) (i.e., having at least 2 cognitive domains 2 SDs below the standardized mean, with ADL deficits). HIV-infected adults with ANI, MND, or HAD comprised the HAND group, while those without cognitive impairment were included in the unimpaired HIV-infected group. Uninfected adults selected for this study did not meet the above criteria for neuropsychological impairment.

\section{MEG cognitive paradigm}

Participants performed 200 total trials of an arrow-based Eriksen flanker task ${ }^{12,13}$ while seated in the magnetically shielded room. Each trial began with a fixation that was presented for 1,450 to 1,550 milliseconds ( $\mathrm{ms}$ ), followed by a row of 5 arrows for 2,500 ms. Participants indicated with their right hand whether the middle arrow was pointing to the left (index finger) or right (middle finger). Trials were pseudorandomized and equally divided between congruent and incongruent conditions, with left and right arrows being equally represented in each of the conditions. Overall MEG recording time for the task was approximately 14 minutes. Using correct trials only, reaction time and accuracy were computed for each condition and were subjected to separate $2 \times 3$ mixed-model analyses of variance (ANOVAs), such that flanker condition (congruent and incongruent) was a within-subjects factor and group (control, unimpaired HIV-infected, and HAND) was a between-subjects factor. Further information on behavioral 
Table Demographics and neuropsychological performance

\begin{tabular}{|c|c|c|c|c|}
\hline & \multicolumn{3}{|c|}{ Group means (SD) } & \multirow[b]{2}{*}{$p$ Value } \\
\hline & Controls & HIV $^{\mathrm{a}}$ & HAND & \\
\hline \multicolumn{5}{|l|}{ Demographics } \\
\hline Age, y & $42.85(6.52)$ & $44.50(8.34)$ & $44.80(7.59)$ & 0.679 \\
\hline Education, y & $16.55(2.11)$ & $15.00(2.79)$ & $15.10(4.96)$ & 0.302 \\
\hline Sex, \% males & 50.00 & 60.00 & 70.00 & 0.453 \\
\hline Handedness, \% right-handed & 90.00 & 95.00 & 90.00 & 0.804 \\
\hline \multicolumn{5}{|l|}{ Neuropsychology } \\
\hline Executive function & $0.16(0.84)$ & $0.05(0.63)$ & $-0.90(0.55)$ & $<0.001$ \\
\hline Verbal fluency & $0.07(1.24)$ & $-0.19(0.89)$ & $-0.85(0.68)$ & 0.004 \\
\hline Semantic fluency & $0.31(1.13)$ & $0.30(1.02)$ & $-0.53(0.91)$ & 0.013 \\
\hline Stroop interference & $0.13(1.15)$ & $-0.48(1.03)$ & $-1.83(1.41)$ & $<0.001$ \\
\hline Trail Making Part B & $0.13(0.92)$ & $0.59(1.00)$ & $-0.43(0.68)$ & 0.002 \\
\hline Attention & $0.26(0.78)$ & $0.06(0.68)$ & $-1.17(0.91)$ & $<0.001$ \\
\hline Symbol search & $0.73(0.73)$ & $0.73(0.83)$ & $-0.43(0.0 .52)$ & $<0.001$ \\
\hline Stroop word & $-0.24(1.13)$ & $-0.61(0.70)$ & $-1.90(1.56)$ & $<0.001$ \\
\hline Speed of processing & $0.10(0.71)$ & $0.22(0.79)$ & $-0.87(0.71)$ & $<0.001$ \\
\hline Digit symbol & $0.10(1.08)$ & $0.52(0.99)$ & $-0.60(0.62)$ & 0.001 \\
\hline Stroop color & $0.02(0.78)$ & $-0.16(0.69)$ & $-1.38(1.40)$ & $<0.001$ \\
\hline Trail Making Part A & $0.23(1.22)$ & $0.30(1.23)$ & $-0.63(0.63)$ & 0.014 \\
\hline \multicolumn{5}{|l|}{ Task performance } \\
\hline Reaction time, ms & $662.77(132.84)$ & $747.38(134.51)$ & $845.13(141.29)$ & $<0.001$ \\
\hline Accuracy in $\%$ correct & $99.13(2.13)$ & 98.95 (2.79) & $96.63(4.96)$ & 0.052 \\
\hline
\end{tabular}

Abbreviation: HAND = HIV-associated neurocognitive disorder.

${ }^{\text {a }}$ HIV denotes HIV-infected group without HAND.

${ }^{\mathrm{b}}$ All neuropsychology scores are $z$ scores, standardized using published normative data (see methods: neuropsychological testing).

analyses can be found in data available from Dryad (appendix e-2, doi.org/10.5061/dryad.1m6764d).

\section{MEG data acquisition}

MEG data were recorded in a 1-layer magnetically shielded room with active shielding engaged to compensate for environmental noise. Neuromagnetic activity was sampled continuously at $1 \mathrm{kHz}$ with an acquisition bandwidth of 0.1 to 330 $\mathrm{Hz}$ using a 306-sensor Elekta system (Helsinki, Finland) equipped with 102 magnetometers and 204 planar gradiometers. Participants were monitored during MEG acquisition by real-time audio-video feeds from inside the room. Each participant's data were individually corrected for head movement and subjected to noise reduction using the temporally extended signal space separation method. ${ }^{14}$ Further details on our MEG data acquisition and analysis methods can be found from data available from Dryad (appendix e-3, doi. org/10.5061/dryad.1m6764d).

\section{MEG data processing, source imaging, and voxel-based statistics}

After data preprocessing, each participant's MEG data were coregistered to their structural T1-weighted MRI data. The MEG data were then split into 2,000-ms epochs ( -500 to 1,500 ), with 0.0 seconds defined as stimulus onset, and transformed into the time-frequency domain. Time-frequency windows of interest were identified using 1-sample $t$ tests on the sensor level spectrograms across all participants, corrected for multiple comparisons using nonparametric permutation testing. We then used a beamforming approach to image cortical activity in the time-frequency windows of interest in each condition per participant. ${ }^{15}$ Briefly, we used a task period and a baseline period of equal duration and bandwidth to compute noise-normalized, source power per voxel. Such source images are commonly referred to as pseudo-t maps, with the unit (pseudo-t) reflecting noise-normalized power differences (i.e., task vs baseline) per voxel. 
To examine neural activity specific to selective attention, source images for the congruent condition were subtracted from the incongruent condition for each participant. This resulted in whole-brain maps of flanker interference activity per participant. We then conducted a whole-brain 1-way ANOVA using each participant's flanker interference map to statistically determine the effects of group. For the ANOVA maps, an a level of 0.05 , a cluster threshold of 500 voxels, and a smoothing kernel of $4 \mathrm{~mm}$ were applied. For follow-up regressions and virtual sensor analyses, we selected the peak voxel with the highest $F$ value for each significant cluster from the 1-way ANOVA of flanker interference activity.

\section{Assessing the relationship between neural activity and neuropsychological measures}

To determine the predictive capacity of neural dynamics for independently measured neuropsychological performance, separate linear regression models were computed for executive function and attention composite scores on flanker interference amplitude values in the voxels of interest (see above). To account for all regions of interest, voxel amplitude measures were entered as stepwise regressions. Ultimately, 4 stepwise regressions were run, split by dependent variable (executive function and attention score) and also by predictors (alpha regions and theta regions). All tests were 2 -tailed, with an $\alpha$ level of 0.05 . All regression analyses were performed in SPSS (Chicago, IL).

\section{Baseline activity analysis}

Recent studies have associated increased spontaneous neural activity with aging. ${ }^{16,17}$ Given theories of accelerated aging in HIV, we examined spontaneous activity during the baseline by performing a voxel time series analysis using our voxels of interest. A baseline value for each participant was calculated by averaging across the entire baseline period within the respective frequency band. Participants with values 2.5 SDs above or below their respective group's mean per region were excluded pairwise. At most, 3 participants were excluded based on these criteria, and in no case were all 3 participants from the same group. For each area of interest, a $2 \times 3$ mixedmodel ANOVA was run on baseline values with a withinsubjects factor of condition (congruent vs incongruent) and a between-subjects factor of group (control, unimpaired HIVinfected, HAND). Conditional effects on baseline values were not expected, but were included as a validity check. Finally, a classification analysis was performed to distinguish unimpaired HIV-infected participants from those with HAND. Details about this discriminant function classification analysis can be obtained from data available from Dryad (appendix e-4, doi.org/10.5061/dryad.1m6764d).

\section{Data availability}

All supplementary data are available from the Dryad Digital Repository: datadryad.org/review?doi=doi:10.5061/dryad.1m6764d. Further anonymized data can be made available to qualified investigators upon request to the corresponding author.

\section{Results}

Briefly, our major findings included slower reaction times and larger flanker interference effects in unimpaired HIV-infected adults and those with HAND. There were also group differences in theta- and alpha-range neural responses in attentionrelated brain regions. In addition, prefrontal theta activity was found to be correlated with attention and executive function composite scores across all participants based on neuropsychological assessment, and to be sharply reduced in those with HAND. Finally, we found HAND-specific differences in spontaneous activity in areas supporting selective attention function.

\section{Participant characteristics}

Neuropsychological and functional assessments indicated that the group with HAND comprised 11 participants with ANI, 6 with MND, and 3 with HAD. The mean duration of HIV diagnosis was 11.7 years in the unimpaired and 12.45 years in the HAND group. The mean $\mathrm{CD} 4^{+} \mathrm{T}$ cell count at the time of the study was 976.00 cells $/ \mathrm{mm}^{3}$ (range: $366-2,617$ ) with an average $\mathrm{CD} 4^{+}$nadir of 275.45 (range: 17-518) in the unimpaired HIV-infected group, while the mean $\mathrm{CD}^{+} \mathrm{T}$ cell count at the time of the study was 783.00 cells $/ \mathrm{mm}^{3}$ (range: $286-1,799$ ) with an average $\mathrm{CD} 4^{+}$nadir of 208.70 (range: 7-440) in the HAND group. The 2 groups did not significantly differ on duration of diagnosis $(p=0.60), \mathrm{CD}^{+}$nadir $(p=0.18)$, or current $\mathrm{CD} 4^{+}$ $(p=0.87)$.

\section{Behavioral selective attention deficit in HIV and HAND}

A mixed-model ANOVA of reaction time revealed a main effect of group $\left(F_{2,57}=9.0, p<0.001\right)$ as well as condition $\left(F_{2,57}=90.2, p<0.001\right)$. There was also a significant groupby-condition interaction $\left(F_{2,57}=8.0, p<0.001\right)$. Post hoc analyses showed that participants with HAND were significantly slower on the task than both unimpaired HIV-infected and control participants $(p<0.05)$. There was also a trend for unimpaired HIV-infected participants to be slower $(p=$ 0.054 ) than controls (figure 1A). To probe the interaction effect, $t$ tests were conducted using the flanker effect data (i.e., the congruent reaction times subtracted from the incongruent reaction times). Participants with HAND had a significantly larger flanker effect compared to both controls (HAND: $124.47 \pm 45.12 \mathrm{~ms}$; control: $44.36 \pm 9.76 \mathrm{~ms} ; p<$ 0.01 ) and unimpaired HIV-infected participants (HIV: $69.46 \pm 16.92 \mathrm{~ms} ; p<0.05$ ), and the unimpaired HIVinfected group also had a significantly larger flanker effect compared to controls ( $p<0.05$; figure $1 \mathrm{~B})$. Finally, we found marginal differences in accuracy by group $(p=0.052)$, whereby participants with HAND had the lowest mean accuracy of $96.63 \%$, unimpaired HIV-infected participants had a mean accuracy of $98.95 \%$, and controls had the highest mean accuracy of $99.13 \%$. However, all 3 groups were more than $95 \%$ accurate, therefore ceiling effects should be considered. 
Figure 1 Behavioral performance distinguishes HIV and HAND status
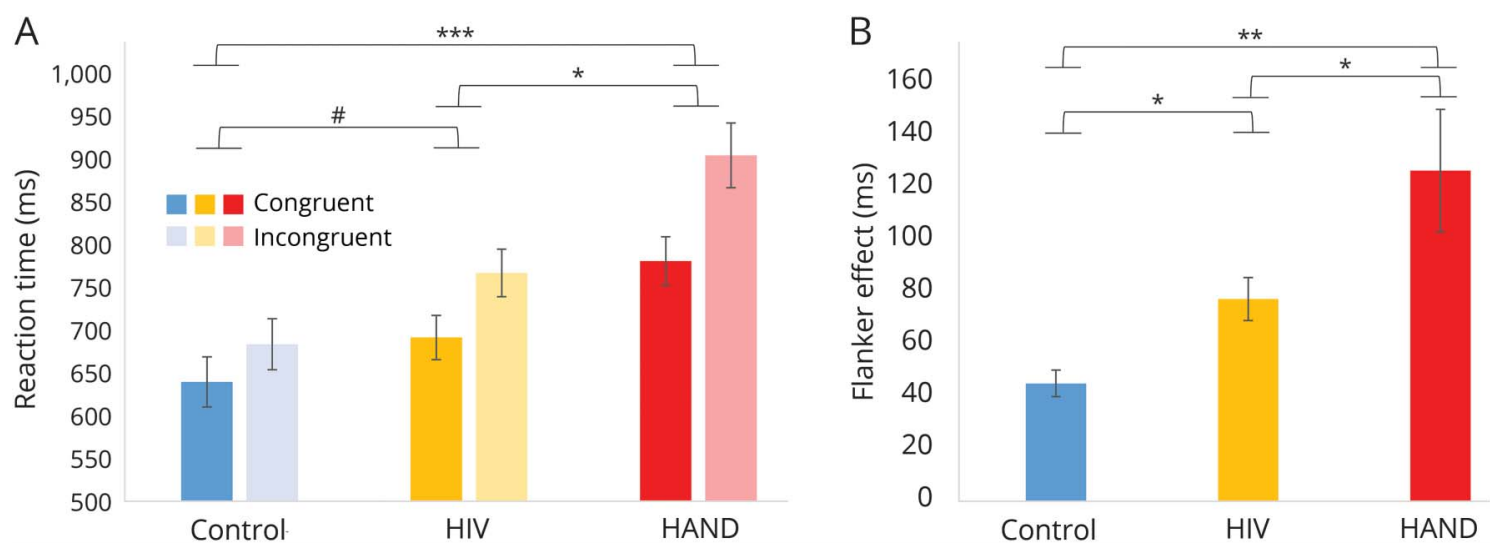

(A) Repeated-measures analysis of variance of reaction time showed significant main effects of condition and group, as well as an interaction effect ( $p<0.001)$. That is, reaction times to the incongruent condition were slower than the congruent condition, participants with HAND had the slowest reaction times, and (B) the HAND group also had the largest difference between congruent and incongruent conditions (i.e., the flanker interference effect). Post hoc significance levels are displayed above the data, with error bars indicating the SEM. ${ }^{*} p<0.10, * p<0.05, * * p<0.01, * \star * p<0.001$. HAND $=$ HIV-associated neurocognitive disorder.

\section{Differential neural activity in HIV and HAND}

In alignment with previous normative studies using the flanker task, ${ }^{13}$ significant responses in the sensor-level data were observed within 2 distinct spectral windows. Specifically, early and transient increases in power were observed in the theta $(3-6 \mathrm{~Hz})$ band between 50 and $400 \mathrm{~ms}$, and decreases in power were observed in the alpha band $(9-12 \mathrm{~Hz})$ between 250 and $650 \mathrm{~ms}$.

To determine the cortical correlates of these responses, these specific time-frequency windows were imaged with a beamforming approach, and flanker interference activity was then calculated by subtracting the congruent condition image from the incongruent condition image for each band separately for each participant. A whole-brain ANOVA of flanker interference activity in the theta range was then conducted, and this showed significant group differences in the left inferior frontal gyrus, left dorsolateral prefrontal cortex (DLPFC), left temporoparietal junction, and the left inferior parietal cortex ( $p<0.05$; figure 2A). Regarding alpha activity, a whole-brain ANOVA of flanker interference activity revealed significant differences in the left superior frontal gyrus, left middle frontal gyrus, left supramarginal gyrus, left angular gyrus, and right DLPFC $(p<0.05$; figure $2 \mathrm{~B})$.

\section{Frontal theta activity is associated with cognitive performance}

To examine the association between the neural markers of selective attention and neuropsychological function, regions of differential flanker interference activity (figure 2) were entered into stepwise regressions with executive function and attention composite scores as the dependent variables. Theta

Figure 2 Group differences for flanker interference activity

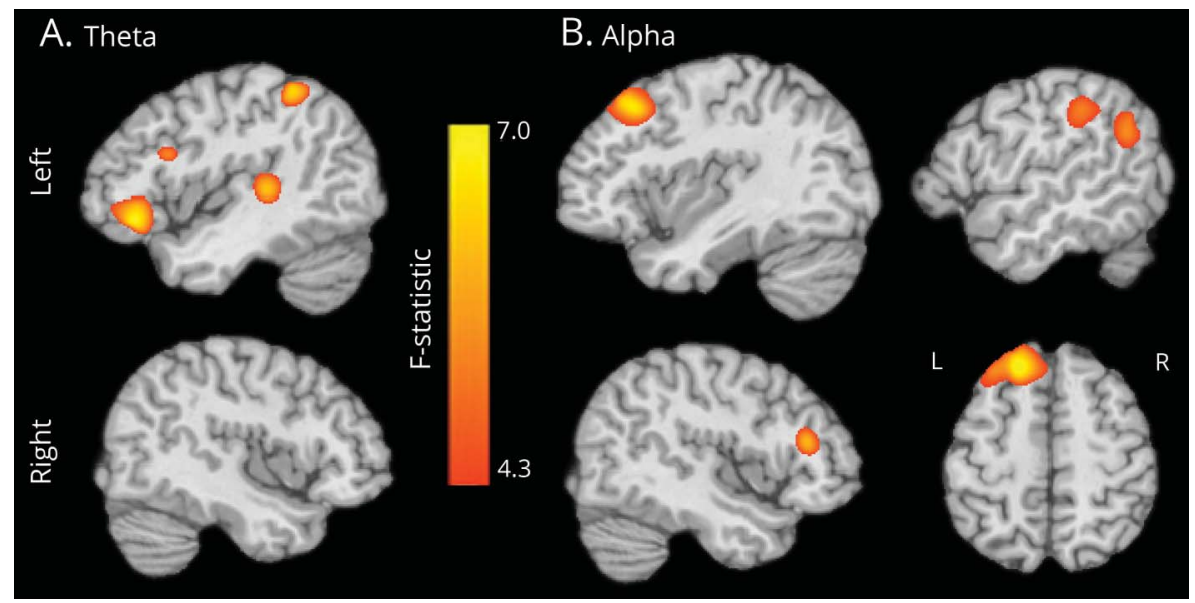

Neural oscillatory activity related to selective attention, defined as flanker interference activity in this study, was calculated for each participant by subtracting neural activity in the congruent condition from that in the incongruent condition. (A) Whole-brain ANOVA of flanker interference activity in the theta range showed significant group differences in the left inferior frontal gyrus, left DLPFC, left temporoparietal junction, and the left inferior parietal cortex. (B) Wholebrain ANOVA of alpha-range flanker interference activity revealed significant differences in the left superior frontal gyrus, left middle frontal gyrus, left supramarginal gyrus, left angular gyrus, and right DLPFC. For both ANOVA maps, an a level of 0.05 ( $F>$ 4.3) and a cluster threshold of 500 voxels were used. ANOVA = analysis of variance; DLPFC = dorsolateral prefrontal cortex. 
range regressions revealed that left inferior frontal activity was a significant positive predictor of executive composite scores $(R=0.34, p=0.009$; figure $3 \mathrm{~A})$, and left DLPFC activity was a significant positive predictor of attention composite scores $(R=0.43, p<0.001$; figure $3 \mathrm{~B})$. The latter association also remained significant when attention and speed of processing domains were combined $(p=0.005)$. No other theta and no alpha regions were significantly associated with attention or executive function scores, or the combined attention/speed of processing composite score. These analyses specifically highlight the relevance of frontal theta activity to selective attention processing and directly link this to neuropsychological performance, which was independently measured.

Since these neuropsychology-related regions also showed group differences in the whole-brain ANOVA, we conducted group-wise post hoc comparisons in both regions and these revealed significant differences between the HAND group and the control and unimpaired HIV-infected groups. That is, theta-band flanker interference activity did not differ between controls and unimpaired HIV-infected participants in either region, but was significantly reduced in those with HAND in both the left inferior frontal and dorsolateral prefrontal cortices (all $p<0.05$; figure 3 ).

\section{Baseline dynamics differ with HIV and HAND status}

Given previous findings linking spontaneous neural activity and aging, we analyzed such activity during the baseline period by extracting virtual sensors from the peak voxels in each significant cluster (figure 2), and averaging across the baseline window. Mixed-model ANOVAs of mean baseline activity in the theta band showed a main effect of group in the left

Figure 3 Frontal theta flanker interference activity predicts neuropsychological performance

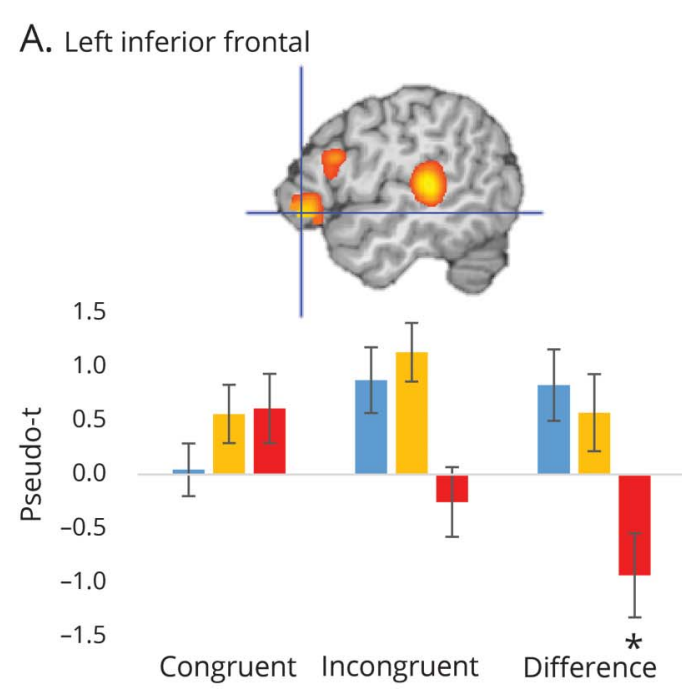

B. Left DLPFC

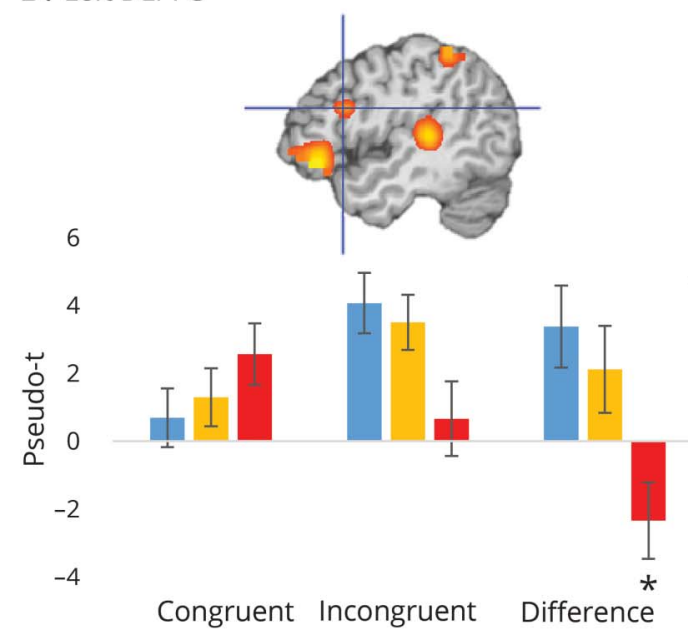

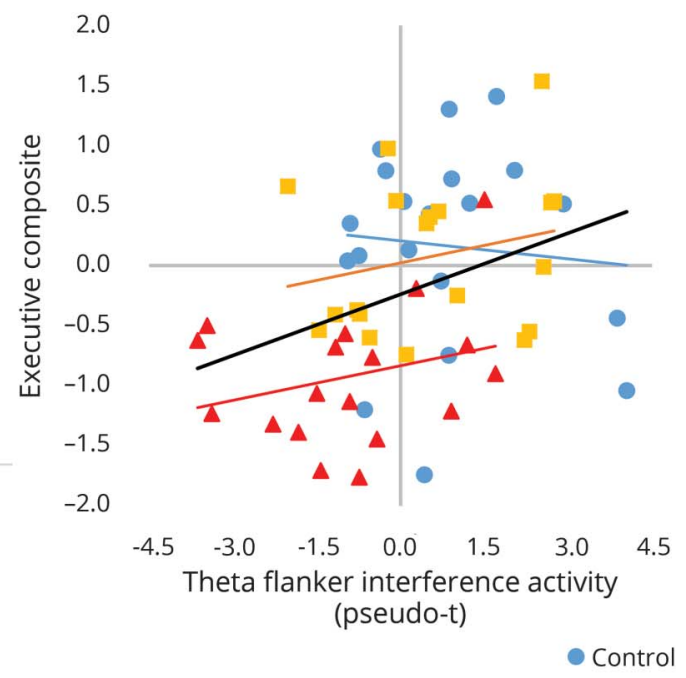

(pseudo-t)

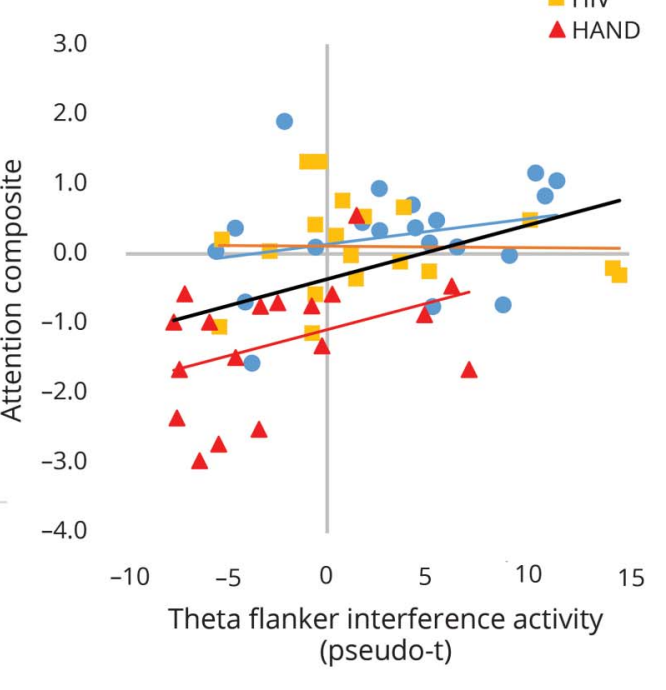

The peak voxel value was extracted from each region identified in the whole-brain analysis of variance maps (figure 2 ) and this was entered into regression analyses with neuropsychological measures. (A) Flanker interference activity in the left inferior frontal gyrus predicted executive function composite scores ( $p$ $<0.05)$, and (B) left DLPFC predicted attention composite scores $(p<0.05)$. ${ }^{\star} p<0.05$ on post hoc significance testing. DLPFC $=$ dorsolateral prefrontal cortex; HAND = HIV-associated neurocognitive disorder. 
inferior frontal gyrus $(p=0.002)$, left DLPFC $(p=0.033)$, and left temporoparietal junction $(p=0.021$; figure 4$)$, and as expected no effect of condition or interaction effect was observed because the activity preceded the stimulus. Post hoc analyses revealed stronger baseline activity in the HAND group compared to matched controls in the left inferior frontal gyrus $(p<0.001)$, left DLPFC $(p=0.011)$, and the left temporoparietal junction $(p=0.016)$. Baseline theta was also stronger in the HAND group compared to the unimpaired HIV-infected group in the left inferior frontal gyrus $(p=$ $0.015)$ and the left temporoparietal junction $(p=0.014)$. Mixed-model ANOVAs of alpha activity during the baseline showed a main effect of group in all voxels of interest $(F>4.4$, $p<0.05$; figure 5), with no condition or interaction effects. Post hoc analyses revealed stronger baseline activity in the HAND group compared to controls in all voxels of interest $(p<0.01)$. In addition, the HAND group had stronger baseline alpha compared to the unimpaired HIV-infected group in the left superior frontal gyrus $(p=0.009)$, left middle frontal gyrus $(p=0.011)$, and right DLPFC $(p=0.009)$. There were also trends for higher baseline alpha activity in the unimpaired HIV-infected group compared to controls in the left supramarginal $(p=0.087)$ and left angular cortex $(p=$ $0.063)$. Finally, an exploratory analysis of HAND subtypes showed increasing spontaneous theta activity with increasing HAND severity in several brain regions, but caution is warranted because of the small subgroup sizes. These interesting results can be obtained from data available from Dryad (figure e-1, doi.org/10.5061/dryad.1m6764d).

Lastly, we conducted exploratory discriminant function analyses to evaluate whether patients with HIV infection could be classified into HAND and non-HAND groups based on spontaneous neural activity metrics. This classification analysis resulted in a significant discriminant model $(p=$ 0.002) showing that baseline theta in the left inferior frontal gyrus and baseline alpha in the left middle frontal gyrus accurately classified patients into HAND and non-HAND groups, with an accuracy of $77.4 \%$ of cross-validated cases. The full model and a more detailed description of the methods can be obtained through data available from Dryad (appendix e-4, doi.org/10.5061/dryad.1m6764d).

\section{Discussion}

In this study, we identified selective attention alterations associated with HIV infection and HAND, and related these behavioral changes to spectrally specific dysfunction across

Figure 4 Spontaneous theta activity is increased in HAND

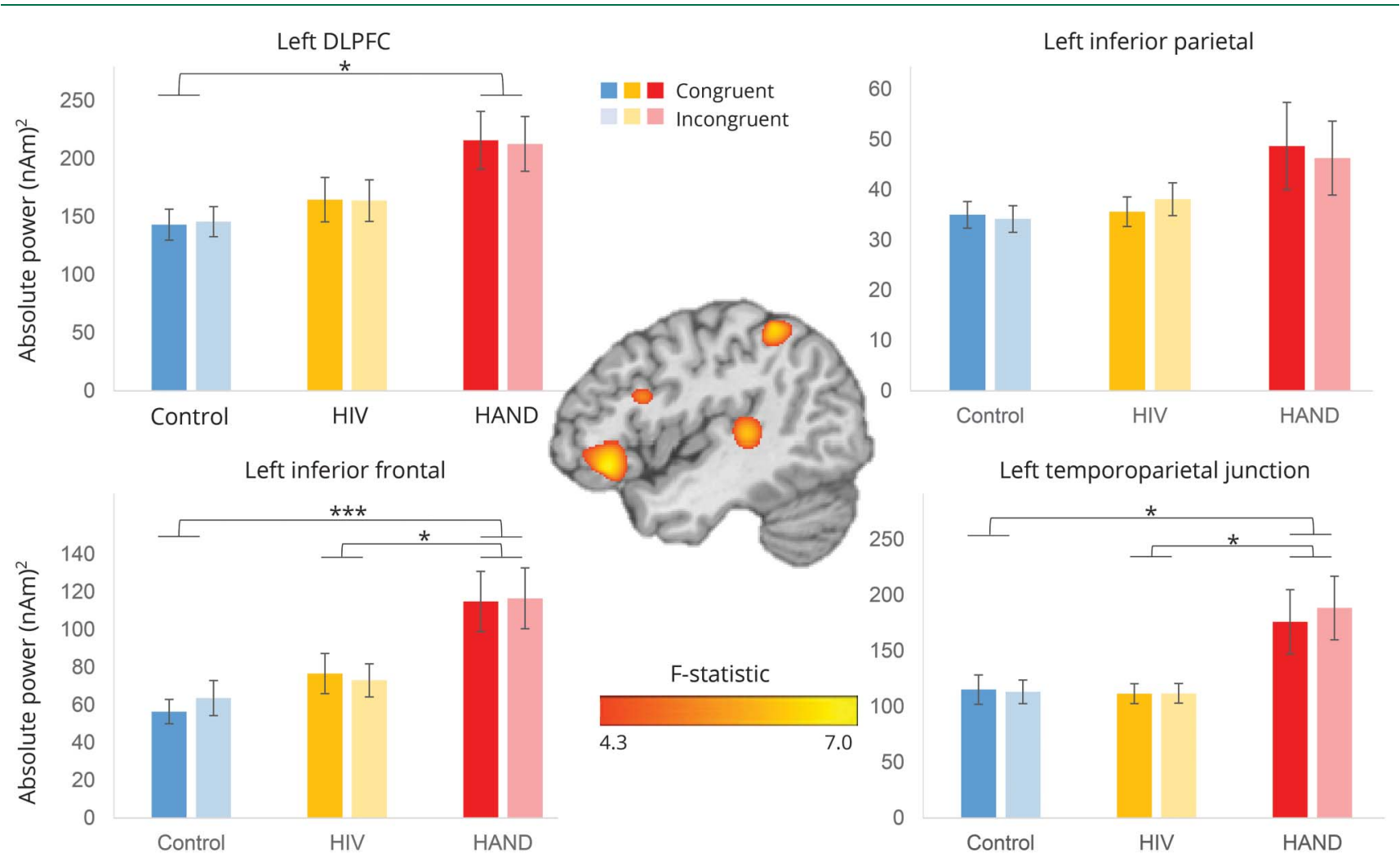

To examine spontaneous neural activity, we performed virtual sensor extraction from the voxels of interest (peak voxel per cluster in figure $2 \mathrm{~A}$ ) for each participant. In each region, the absolute power was then averaged across the baseline period in the resulting time series of each participant. Repeatedmeasures analysis of variance showed a significant main effect of group in 3 of the 4 areas of interest $(p<0.05)$. The results of post hoc significance tests are displayed above the data, with error bars indicating the SEM. ${ }^{\star} p<0.05,{ }^{*} * p<0.001$. DLPFC $=$ dorsolateral prefrontal cortex; HAND $=$ HIV-associated neurocognitive disorder. 

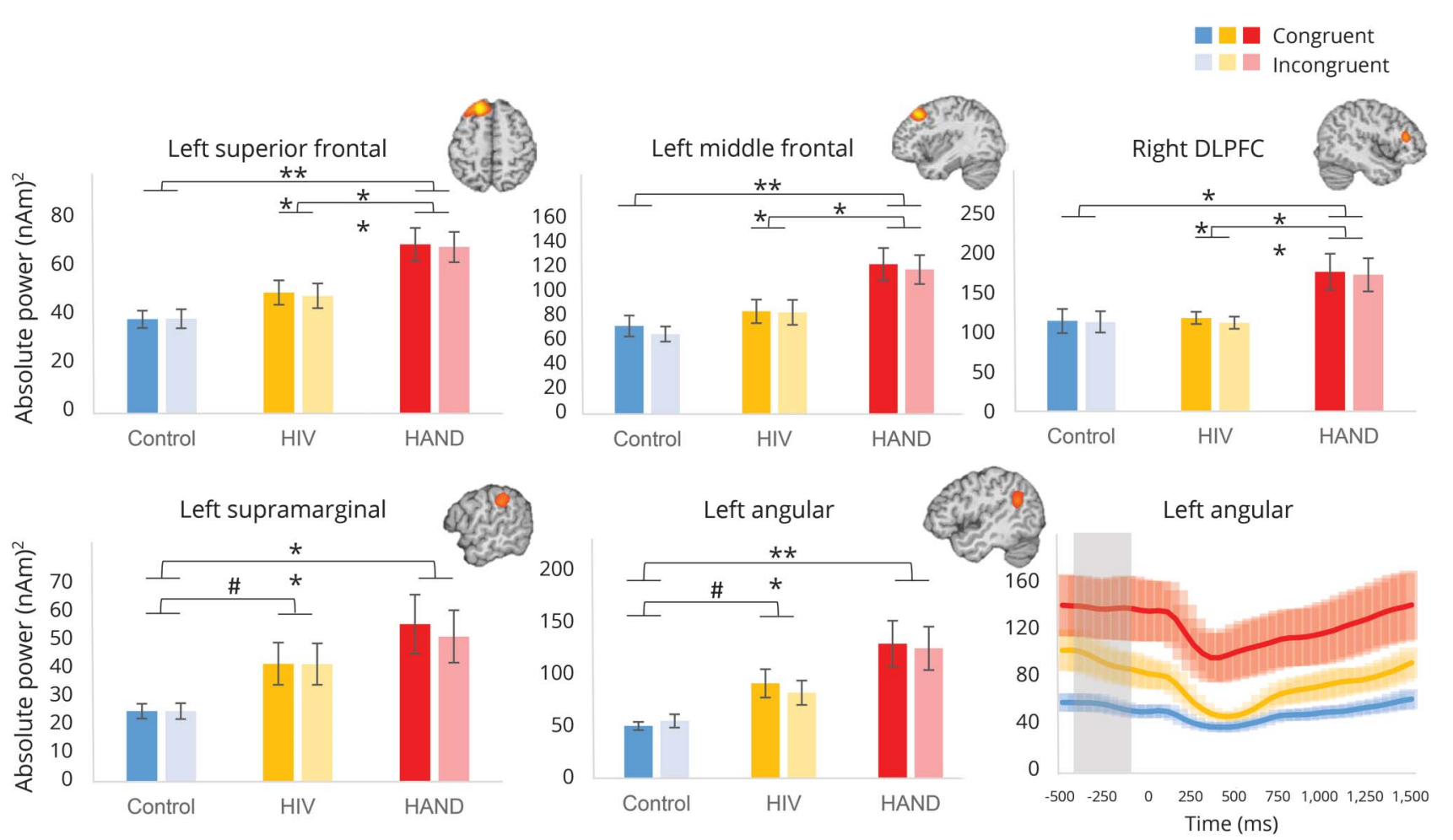

Virtual sensor analysis for the alpha band was also performed using the peak voxels from figure $2 \mathrm{~B}$. An average of the absolute power per region was calculated across the baseline for each participant. Repeated-measures analysis of variance showed a significant main effect of group in all 5 areas of interest $(p<0.05)$. The results of post hoc significance tests are displayed above the bars. An example group-averaged time series of the entire epoch from the left angular gyrus is also displayed, with the gray bar indicating the baseline time interval. Error bars indicate the SEM. ${ }^{*} p<0.10,{ }^{\star} p<0.05,{ }^{\star *} p<0.01,{ }^{\star \star \star} * p<0.001$. $\mathrm{DLPFC}=$ dorsolateral prefrontal cortex; HAND = HIV-associated neurocognitive disorder.

multiple cortical areas. Critically, flanker-related theta activity in the unimpaired HIV-infected and HAND groups differed in the left inferior frontal gyrus and DLPFC, and this activity was correlated with performance on neuropsychological testing of executive function and attention, respectively. In addition, participants with HAND also had altered spontaneous activity in all brain areas where there were selective attention group effects, with the exception of theta activity in the inferior parietal cortex. Higher spontaneous theta and alpha activity was not only associated with HAND, but also increased in a stepwise fashion from controls to unimpaired HIV-infected to HAND in several brain regions. Studies examining the neural dynamics of selective attention in HIV are rare, and, of note, our study uniquely examines this in participants with HAND separately from unimpaired HIV-infected participants. These findings are important not only in characterizing the neural dynamics of impaired selective attention in participants with HAND, but also in progressing toward a potential presymptomatic marker of neural alterations associated with HIV infection.

Impaired attention function is an important component of cognitive dysfunction in HIV-infected adults as, ${ }^{18}$ even in the cART era, attention deficits remain prevalent. ${ }^{19,20}$ However, we still have a limited understanding of the neural origin of these deficits and the associated mechanisms. Previous neuroimaging studies using fMRI have shown HIV-related increases in activation in task-specific brain areas serving attention and working memory (e.g., parietal and prefrontal cortices), ${ }^{6,7,21-24}$ although these studies did not distinguish HIV-infected participants based on HAND status and did not examine the temporal dynamics. Electrophysiologic methods such as EEG and MEG have also been emerging as powerful tools for identifying HIV-related cortical dysfunction, ${ }^{25-27}$ and have identified abnormal prefrontal and parietal activity during multiple cognitive tasks. ${ }^{8,9,28-31}$ Of note, such studies have also shown that MEG metrics have high test-retest reliability in HIV-infected and uninfected participants, which of course is critical for any potential marker of disease. ${ }^{32}$ Therefore, examining neuroHIV through the lenses of attention function and MEG may provide specific and reliable systems-level insight into the resulting cognitive deficits.

Neurobehaviorally, our reaction time analyses of the flanker selective attention task revealed group, condition, and interaction effects, ultimately showing that the behavioral flanker effect was increased in participants with HIV, with the most accentuated effects in those with HAND. This larger flanker effect reflects progressive deficits in selective attention function from controls to unimpaired HIV-infected to HAND 
participants. While larger flanker effects in HIV-infected individuals has been previously reported, ${ }^{33}$ HAND status was not taken into account. Thus, the current data demonstrate impaired selective attention processing on a neurobehavioral level, even in patients without clinically significant symptoms.

Group theta differences in flanker interference activity were found in various cortical areas associated with attention processing. For example, the inferior parietal lobule, DLPFC, temporoparietal junction, and inferior frontal gyrus have all been shown to be involved in attention processing. ${ }^{34-37}$ Of note, a meta-analysis of fMRI studies of neuroHIV showed reliable differences in the left inferior frontal gyrus between HIV-infected and control groups. ${ }^{38}$ Thus, our results expand on this finding by showing that aberrations in this area are predictive of executive function on neuropsychological testing, and that the actual deficit appears to be limited to neuronal responses in the theta range. That is, in HIV and HAND, decreasing theta activity in the left inferior frontal gyrus was associated with decreased executive function performance on independent tests. In addition, decreasing theta activity in the left DLPFC was associated with decreased attention performance (and decreased attention + speed of processing). These associations between task-related neural responses and separately measured neuropsychological testing highlight the relevance of frontal theta activity in neuroHIV and HAND.

Group alpha differences were also found in regions relevant to attention processing ${ }^{39,40}$; however, alpha activity in those regions was not found to be associated with neuropsychological function. Nonetheless, our findings do broadly implicate aberrant alpha activity in HIV infection, and this is consistent with previous studies. Specifically, HIV-related alpha differences have been reported in the left supramarginal gyrus with respect to a working memory task, ${ }^{9}$ and in the right DLPFC during a simple visual processing task. ${ }^{8}$ Both of these brain regions exhibited differential alpha activity in participants with HIV infection. Furthermore, our investigation of spontaneous neural activity during the baseline also revealed significant HIV-related alterations in alpha.

Examination of spontaneous cortical activity revealed that baseline levels of neural activity were altered in HIV-infected participants, and that this activity exhibited a stepwise increase. That is, controls had the lowest levels of spontaneous theta and alpha activity, unimpaired HIV-infected participants had increased levels, and those with HAND had the highest levels of spontaneous activity during the baseline. Of note, this pattern of increased baseline activity was seen across all regions of interest in both theta and alpha bands, although it was not significant for theta in the left inferior parietal and not every stepwise comparison between unimpaired HIV-infected and control groups was significant. Nonetheless, the stability of the overall pattern across a large number of cortical regions serving selective attention was striking, and suggests that there may be reliable differences due to HIV disease even before any sign of cognitive deficit. Such markers would be invaluable and potentially allow the objective identification of patients who are likely to develop HAND very early in the disease process. Our exploratory classification analysis based on the power of spontaneous activity was an initial step toward this long-range goal, and it provided preliminary evidence that such classification is possible, but future studies are needed to test the algorithm on an independent sample. Of note, elevated spontaneous neural activity has also been reported in the aging literature, ${ }^{16,17}$ and one recent study showed elevated spontaneous activity in the occipital cortices of HIV-infected participants with and without HAND. ${ }^{10}$ Previous studies have also drawn connections between HIV-related neurocognitive changes and accelerated aging. ${ }^{41,42}$ Given that our middleaged sample was closely matched on age, these findings may lend additional support to the idea of accelerated aging in neuroHIV.

Before closing, it is important to reflect on some of the limitations of this study. First, our sample size regarding the HAND group was ample, but not large enough to be subdivided into asymptomatic, mild, and HIV-associated dementia subgroups. We did, however, qualitatively note an increase in spontaneous theta activity with progression from ANI, to MND, to HAD. Our sample size was also not large enough to adequately test our classification algorithm. Future analyses using larger, independent samples should further examine such baseline differences in neuroHIV, as well as further validate the use of these measures for accurate classification of HAND. Another possible limitation was that the difficulty of our flanker task was low, such that the accuracy of each group remained above $96 \%$. This ceiling effect led us to focus our analysis on reaction times. More complex attention tasks would not only allow the investigation of other aspects of attention, but might also allow for more variability in accuracy on the task. Lastly, we did not examine sex differences or the effect of cART in this study, and future work should evaluate whether these factors modulate some of the effects we observed.

In summary, we found spectrally specific neural changes in neuroHIV using state-of-the-art MEG imaging. By splitting our HIV-infected group by HAND status, we were able to tease apart important differences between these groups. Our main findings were 2 -fold. First, we found alterations in selective attention measures (both behavioral and neural) in otherwise cognitively normal HIV-infected participants. Such metrics may hold promise for predicting the onset of cognitive deficits before clinical symptoms arise, which would enhance prognostic abilities and allow for more sensitive monitoring of disease progression and treatment effects. Second, we observed even greater impairment in individuals diagnosed with HAND, highlighting the relationship between our neural measures and clinical outcome. Specifically, decreased theta activity in the left inferior frontal cortex and left DLPFC was associated with decreased executive and attention function, respectively. In the future, these measures may be useful in 
predicting HAND and/or the trajectory of cognitive decline, but further research is needed. Future studies should further evaluate these metrics and test the specificity of selective attention tasks vs other demanding neurocognitive tests for eliciting dysfunction in neuroHIV.

\section{Author contributions}

B.J. Lew: study concept and design, analysis and interpretation of data, statistical analysis, drafting manuscript for intellectual content. T.J. McDermott: analysis and interpretation of data. A.I. Wiesman: analysis and interpretation of data. J. O’Neill: study concept and design. M.S. Mills: acquisition of data, analysis of data. K.R. Robertson: study concept and design, revising manuscript for intellectual content. H.S. Fox: study concept and design. S. Swindells: study concept and design, revising manuscript for intellectual content, study supervision. T.W. Wilson: study concept and design, revising manuscript for intellectual content, study supervision.

\section{Study funding}

Supported by NIH (MH103220, MH116782, MH062261, AG055332), NSF (1539067), and by a Research Support Fund grant from the Nebraska Health System and the University of Nebraska Medical Center.

\section{Disclosure}

The authors report no disclosures relevant to the manuscript. Go to Neurology.org/ $\mathrm{N}$ for full disclosures.

\section{Publication history}

Received by Neurology February 26, 2018. Accepted in final form August 2, 2018.

\section{References}

1. Antinori A, Arendt G, Becker JT, et al. Updated research nosology for HIV-associated neurocognitive disorders. Neurology 2007;69:1789-1799.

2. Clifford DB, Ances BM. HIV-associated neurocognitive disorder. Lancet Infect Dis 2013;13:976-986.

3. Heaton RK, Franklin DR, Ellis RJ, et al. HIV-associated neurocognitive disorders before and during the era of combination antiretroviral therapy: differences in rates, nature, and predictors. J Neurovirol 2011;17:3-16.

4. Eggers C, Arendt G, Hahn K, et al. HIV-1-associated neurocognitive disorder: epidemiology, pathogenesis, diagnosis, and treatment. J Neurol 2017;264:1715-1727.

5. Ances BM, Vaida F, Yeh MJ, et al. HIV infection and aging independently affect brain function as measured by functional magnetic resonance imaging. J Infect Dis 2010; 201:336-340.

6. Chang L, Speck O, Miller EN, et al. Neural correlates of attention and working memory deficits in HIV patients. Neurology 2001;57:1001-1007.

7. Chang L, Yakupov R, Nakama H, Stokes B, Ernst T. Antiretroviral treatment is associated with increased attentional load-dependent brain activation in HIV patients. J Neuroimmune Pharmacol 2008;3:95-104.

8. Wilson TW, Fox HS, Robertson KR, et al. Abnormal MEG oscillatory activity during visual processing in the prefrontal cortices and frontal eye-fields of the aging HIV brain. PLoS One 2013;8:e66241.

9. Wilson TW, Proskovec AL, Heinrichs-Graham E, et al. Aberrant neuronal dynamics during working memory operations in the aging HIV-infected brain. Sci Rep 2017;7: 41568 .

10. Wiesman AI, O’Neill J, Mills MS, et al. Aberrant neural dynamics during visuospatial perception differentiate HIV-infection and HAND. Brain 2018;141:1678-1690.

11. Heaton RK, Miller SW, Taylor MJ, Grant I. Revised Comprehensive Norms for an Expanded Halstead-Reitan Battery: Demographically Adjusted Neuropsychological Norms for African American and Caucasian Adults. Lutz: Psychological Assessment Resources; 2004
12. Eriksen BA, Eriksen CW. Effects of noise letters upon the identification of a target letter in a nonsearch task. Percept Psychophys 1974;16:143-149.

13. McDermott TJ, Wiesman AI, Proskovec AL, Heinrichs-Graham E, Wilson TW. Spatiotemporal oscillatory dynamics of visual selective attention during a flanker task. Neuroimage 2017; 156:277-285.

14. Taulu S, Simola J. Spatiotemporal signal space separation method for rejecting nearby interference in MEG measurements. Phys Med Biol 2006;51:1759-1768.

15. Gross J, Kujala J, Hamalainen M, Timmermann L, Schnitzler A, Salmelin R. Dynamic imaging of coherent sources: studying neural interactions in the human brain. Proc Natl Acad Sci USA 2001;98:694-699.

16. Spooner RK, Wiesman AI, Proskovec AL, Heinrichs-Graham E, Wilson TW. Rhythmic spontaneous activity mediates the age-related decline in somatosensory function. Cereb Cortex Epub 2018 Jan 12.

17. Heinrichs-Graham E, Wilson TW. Is an absolute level of cortical beta suppression required for proper movement? Magnetoencephalographic evidence from healthy aging. Neuroimage 2016;134:514-521.

18. Woods SP, Moore DJ, Weber E, Grant I. Cognitive neuropsychology of HIVassociated neurocognitive disorders. Neuropsychol Rev 2009;19:152-168.

19. Cysique LA, Brew BJ. Neuropsychological functioning and antiretroviral treatment in HIV/AIDS: a review. Neuropsychol Rev 2009;19:169-185.

20. Sacktor N, Skolasky RL, Seaberg E, et al. Prevalence of HIV-associated neurocognitive disorders in the Multicenter AIDS Cohort Study. Neurology 2016;86:334-340.

21. Chang L, Tomasi D, Yakupov R, et al. Adaptation of the attention network in human immunodeficiency virus brain injury. Ann Neurol 2004;56:259-272.

22. Ernst T, Chang L, Jovicich J, Ames N, Arnold S. Abnormal brain activation on functional MRI in cognitively asymptomatic HIV patients. Neurology 2002;59: 1343-1349.

23. Ernst T, Yakupov R, Nakama H, et al. Declined neural efficiency in cognitively stable human immunodeficiency virus patients. Ann Neurol 2009;65:316-325.

24. Hakkers CS, Arends JE, Barth RE, Du Plessis S, Hoepelman AI, Vink M. Review of functional MRI in HIV: effects of aging and medication. J Neurovirol 2017;23:20-32.

25. Becker JT, Bajo R, Fabrizio M, et al. Functional connectivity measured with magnetoencephalography identifies persons with HIV disease. Brain Imaging Behav 2012; 6:366-373.

26. Becker JT, Cuesta P, Fabrizio M, et al. Brain structural and functional recovery following initiation of combination antiretroviral therapy. J Neurovirol 2012;18: 423-427.

27. Fernandez-Cruz AL, Fellows LK. The electrophysiology of neuroHIV: a systematic review of EEG and MEG studies in people with HIV infection since the advent of highly-active antiretroviral therapy. Clin Neurophysiol 2017;128:965-976.

28. Becker KM, Heinrichs-Graham E, Fox HS, et al. Decreased MEG beta oscillations in HIV-infected older adults during the resting state. J Neurovirol 2013;19:586-594.

29. Wilson TW, Heinrichs-Graham E, Becker KM, et al. Multimodal neuroimaging evidence of alterations in cortical structure and function in HIV-infected older adults. Hum Brain Mapp 2015;36:897-910.

30. Wilson TW, Heinrichs-Graham E, Proskovec AL, McDermott TJ. Neuroimaging with magnetoencephalography: a dynamic view of brain pathophysiology. Transl Res 2016;175:17-36.

31. Wilson TW, Heinrichs-Graham E, Robertson KR, et al. Functional brain abnormalities during finger-tapping in HIV-infected older adults: a magnetoencephalography study. J Neuroimmune Pharmacol 2013;8:965-974.

32. Becker JT, Fabrizio M, Sudre G, et al. Potential utility of resting-state magnetoencephalography as a biomarker of CNS abnormality in HIV disease. J Neurosci Methods 2012;206:176-182.

33. Wang YQ, Pan Y, Zhu S, Wang YG, Shen ZH, Wang K. Selective impairments of alerting and executive control in HIV-infected patients: evidence from attention network test. Behav Brain Funct 2017;13:11.

34. Heinen K, Feredoes E, Ruff CC, Driver J. Functional connectivity between prefrontal and parietal cortex drives visuo-spatial attention shifts. Neuropsychologia 2017;99:81-91.

35. Hopfinger JB, Buonocore MH, Mangun GR. The neural mechanisms of top-down attentional control. Nat Neurosci 2000;3:284-291.

36. Ptak R. The frontoparietal attention network of the human brain: action, saliency, and a priority map of the environment. Neuroscientist 2012;18:502-515.

37. Swick D, Ashley V, Turken AU. Left inferior frontal gyrus is critical for response inhibition. BMC Neurosci 2008;9:102.

38. Plessis SD, Vink M, Joska JA, Koutsilieri E, Stein DJ, Emsley R. HIV infection and the fronto-striatal system: a systematic review and meta-analysis of fMRI studies. AIDS 2014;28:803-811.

39. Lobier M, Palva JM, Palva S. High-alpha band synchronization across frontal, parietal and visual cortex mediates behavioral and neuronal effects of visuospatial attention. Neuroimage 2018;165:222-237.

40. Suzuki K, Okumura Y, Kita Y, Oi Y, Shinoda H, Inagaki M. The relationship between the superior frontal cortex and alpha oscillation in a flanker task: simultaneous recording of electroencephalogram (EEG) and near infrared spectroscopy (NIRS). Neurosci Res 2018;131:30-35.

41. Levine AJ, Quach A, Moore DJ, et al. Accelerated epigenetic aging in brain is associated with pre-mortem HIV-associated neurocognitive disorders. J Neurovirol 2016; 22:366-375.

42. Sheppard DP, Iudicello JE, Morgan EE, et al. Accelerated and accentuated neurocognitive aging in HIV infection. J Neurovirol 2017;23:492-500. 


\section{Neurology}

\section{Neural dynamics of selective attention deficits in HIV-associated neurocognitive disorder}

Brandon J. Lew, Timothy J. McDermott, Alex I. Wiesman, et al.

Neurology 2018;91;e1860-e1869 Published Online before print October 17, 2018

DOI 10.1212/WNL.0000000000006504

This information is current as of October 17, 2018

\section{Updated Information \&} Services

References

Subspecialty Collections

Permissions \& Licensing

\section{Reprints}

including high resolution figures, can be found at: http://n.neurology.org/content/91/20/e1860.full

This article cites 40 articles, 5 of which you can access for free at: http://n.neurology.org/content/91/20/e1860.full\#ref-list-1

This article, along with others on similar topics, appears in the following collection(s):

All clinical neurophysiology

http://n.neurology.org/cgi/collection/all_clinical_neurophysiology Assessment of cognitive disorders/dementia

http://n.neurology.org/cgi/collection/assessment_of_cognitive_disorder s_dementia

Attention

http://n.neurology.org/cgi/collection/attention

HIV dementia

http://n.neurology.org/cgi/collection/hiv_dementia

Information about reproducing this article in parts (figures,tables) or in its entirety can be found online at:

http://www.neurology.org/about/about_the_journal\#permissions

Information about ordering reprints can be found online:

http://n.neurology.org/subscribers/advertise

Neurology ${ }^{\circledR}$ is the official journal of the American Academy of Neurology. Published continuously since 1951 , it is now a weekly with 48 issues per year. Copyright Copyright (C) 2018 The Author(s). Published by Wolters Kluwer Health, Inc. on behalf of the American Academy of Neurology.. All rights reserved. Print ISSN: 0028-3878. Online ISSN: 1526-632X.

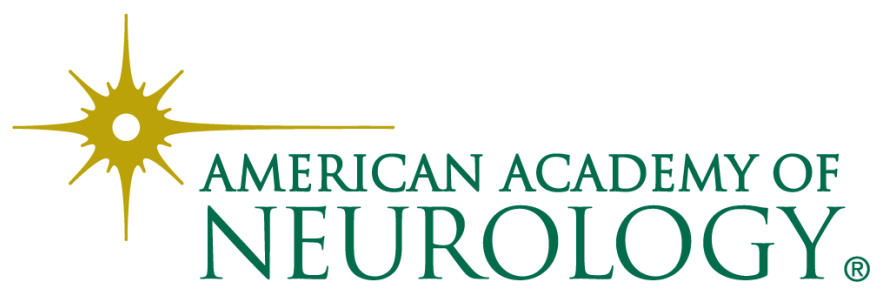

